\title{
STRESS AND SENSITIVITY ANALYSIS OF CANTILEVER BASED MEMS SENSOR FOR ENVIRONMENTAL APPLICATIONS
}

\author{
A. Nallathambi ${ }^{1}$ and T. Shanmuganantham ${ }^{2}$ \\ ${ }^{1}$ Research Scholar, Department of Electronics Engineering, Pondicherry University, Pondicherry, India. \\ ${ }^{2}$ Assistant Professor, Department of Electronics Engineering, Pondicherry University, Pondicherry, India. \\ \{1'nallathambiarjunan@gmail.com, ${ }^{2}$ shanmuga.dee@pondiuni.edu.in\}
}

\begin{abstract}
We present a cantilever based Micro-electromechanical System (MEMS) humidity sensor for applications of environmental monitoring. The main aim of this paper is to design, simulate and analyze the micro cantilever based MEMS sensor such as V, $\mathrm{U}$ and $\mathrm{W}$ shaped cantilever using Polyimide sensing material. The performance of metrics such as the maximum induced stress, deflection and sensitivity obtained through the finite element tool have been analyzed using the simulation software INTELLISUITE v8.7. A designed Si cantilever humidity sensor based on mechanical principles of displacement analysis is being performed on the sensor. The sensing material as Polyimide is coated over the beam to sense the humidity. When the humidity absorbed by the Polyimide material, the mass of the beam is also increased. The change in humidity element is bending of the micro cantilever that modifies the measured displacement between the substrate and the micro cantilever. With this change in displacement gives the measure of the amount of water vapor present in that environment. Therefore the result of these device studies can be used to enhance the sensitivity and stress. Finally, we observed that the best output displacement, Mises stress and sensitivity responses are obtained from W shaped cantilever in the range of $10 \% \mathrm{RH}$ to $100 \% \mathrm{RH}$.
\end{abstract}

Keywords: MEMS, Cantilever, Displacement, Stress and Sensitivity

\section{Introduction}

The recent MEMS researches focus on several concentrating areas of science and technology such as health sciences and biotechnology. MEMS devices have been used in many applications such as environmental monitoring, electronics, agriculture and biomedical fields. A cantilever is some of the earliest and most elementary MEMS devices. A cantilever is fixed at one end and the other end is free to move when it experiences some stress. MEMS device used to detect the physical, chemical or biological effects when the changes in cantilever bending or vibrational frequency. Nickolay V. Lavrik, et al. [1] has been discussed both conventional and cantilever sensors are proposed to greatly reduce the size, high precision, improve dynamic response and increased reliability. Chatzandroulis et al. [2] have been obtained two basic principles of operation. One is a cantilever with stress induced Piezo-resistive readout, covered with a water absorbing polymer layer, and another one is capacitive type devices where transduction is accomplished by monitoring the change in dielectric constant of a hydrophilic polymer. B. Gardiner et al. [3] have been developed applications in a cyberspace of things and wireless sensor networks require sensing of environmental and multiple physical parameters. In Japan physical and environmental monitoring systems are used to measure gamma and beta radiation levels and weather conditions. Anja Boisen et al. [4] have been focused on the cantilever design of geometry, but will include a few related beam-based sensors with the same sensing principles. Hamid Farahani, et al. [5] was developed recent humidity sensors in both industry and university research, provide promising performance with the high impact contribution to an improvement of accuracy, reliability, economic efficiency and especially those incorporated with nano-scale elements. Lung-Tai Chen et al. [6] have obtained the proposed device sensing mechanism was based on a composite cantilever structure containing a Platinum (Pt) resistor accumulated on a Silicon nitride $\left(\mathrm{Si}_{3} \mathrm{Ni}_{4}\right)$ membrane and coated by a Polyimide sensing layer. Brugger et al. [7] have been pointed out that the cantilever sensor is the uncomplicated device among MEMS devices that overtures a very promising for forthcoming development such as novel physical, chemical and biological sensors. A.Nallathambi et al. [8-9] have been analyzed and compared the various microcantilever parameters of the performance like deflection, output displacement and sensitivity. The various cantilever parameters are adequate according to the requirements of the application for optimizing the performance. Rectangular, T-shaped and V-shaped cantilevers are the most commonly used cantilever geometry for sensors. In this work, we enhance and develop the $\mathrm{W}$ shaped cantilever to get an enhanced sensitivity and stress using the difference in the dimensions of a micro cantilever.

In this paper described studies on various humidity sensor designed methodology, principles of materials, fabrication technologies, moisture sensing mechanisms and applications. Further, new dimensions and developments of sensitive materials, i.e., composite 
cantilever or single-species in the nano scale processing system which among the principal considerations. The proposed MEMS sensor three different types of micro devices, namely a V, U and $\mathrm{W}$ shape cantilever based micro humidity sensor. MEMS cantilever type humidity sensor, which absorb a micro cantilever covered on one side with a water-absorbent layer. This can be absorbed a great potential for high sensitivity, high stability and high linearity. This sensor in moist conditions, water is absorbed by sensing layer and this principle a variation in the cantilever surface stress or in the mass changes from which the humidity can be determined.

\section{Humidity Sensor Design and Methodolgy}

Humidity is described as the measure of water vapour in an atmosphere of air or other gases. To present design the pressure equivalent of humidity is taken into consideration as an experimental value of temperature at $30^{\circ} \mathrm{C}$ and it is subjected to cantilever structure for measurement of the output deflection. In present cases relative humidity is subject to cantilever beam which results of deflection. In this work, consider the pressure equivalent of deflection is obtained from the relative humidity saturation pressure [4]. The deflection of cantilever beam is given by the relation as an equation (1),

$$
\delta=3 \times \sigma_{s} \times \frac{(1-v)}{E} \subset(L / t)^{2}
$$

Where E, v are the Young's Modulus and the poisson's ratio, $\mathrm{L}$ is beam length, $\mathrm{t}$ is thickness of cantilever and

$$
K=\frac{F}{\delta}=\frac{E W t^{3}}{4 L^{3}}
$$

The equation (2) relates the spring constant $\mathrm{K}$ to the cantilever dimensions and material constants, then F, W are the force and width of the cantilever.

The deformation of the V, U and W Shape cantilever depends upon the length (L), width (W) and thickness (t) of the dimensions and it also depends on different properties of the material used to design the cantilever. Mention the above equations determine that the output displacement is similar to the realized pressure. Therefore the sensitivity of the cantilever sensor when changes the dimension with the modification of sensing material and also shape of the cantilever. The study is being completed on the different types of design structure with the successive dimensions which is shown in the table 1. Thus the surface stress of the water vapor can be analysed to the output displacement value.

The construction of various shapes with cantilever determines the stiffness of the cantilever by using analytical design of the cantilever beam and also the properties of the sensing material. So that the sensitivity of the cantilever beam changes with using the sensing material. The study is being done on the design of a structure with the following materials and dimensions which are shown in the table 1 . The Humidity sensor model is drawn by using the $3 \mathrm{D}$ builder module of the
Intellisuite software. Figure 1, 2 and 3 shows the V, U and $\mathrm{W}$ micro cantilever structure. In the proposed design, we used Polyimide as sensing materials of humidity sensors.

In first model V shape cantilever is used with Polyimide as sensing layer and Silicon-dioxide as dielectric layer. Because

Table1: Parameter values of Cantilever

\begin{tabular}{|c|c|c|c|c|}
\hline $\begin{array}{c}\text { Layer/ } \\
\text { Material }\end{array}$ & $\begin{array}{c}\text { Dimensions } \\
{[\mu \mathrm{m}]}\end{array}$ & $\begin{array}{c}\text { Young's } \\
{[\mathrm{GPa}]}\end{array}$ & Poisson's & $\begin{array}{c}\text { Density } \\
{\left[\mathrm{q} / \mathrm{cm}^{3}\right]}\end{array}$ \\
\hline $\begin{array}{c}\text { Substrate/ } \\
\mathrm{Si}\end{array}$ & $500 \times 200 \times 10$ & 170 & 0.26 & 2.3 \\
\cline { 1 - 2 } $\begin{array}{c}\text { Structural/ } \\
\mathrm{Si}\end{array}$ & $400 \times 20 \times 2.4$ & 296 & 0.275 & 3.1 \\
\hline $\begin{array}{c}\text { Functional } \\
/ \mathrm{Si}_{3} \mathrm{~N}_{4}\end{array}$ & $400 \times 20 \times 2$ & 160 & 0.226 & 2.3 \\
\hline $\begin{array}{c}\text { Sensing/ } \\
\text { Polyimide }\end{array}$ & $400 \times 20 \times 0.5$ & & & \\
\hline
\end{tabular}

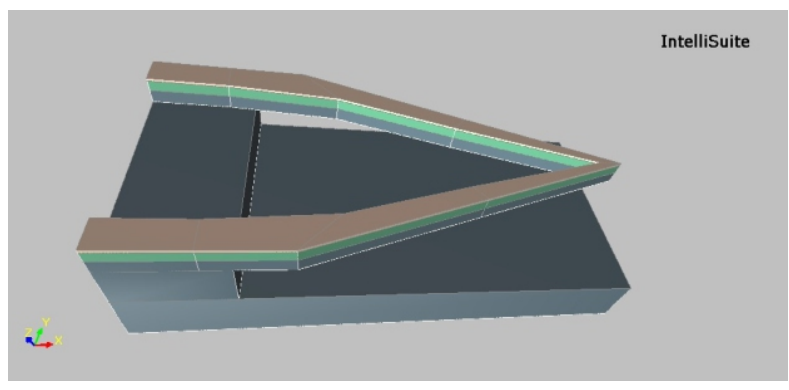

Fig. 1 V Shaped Cantilever

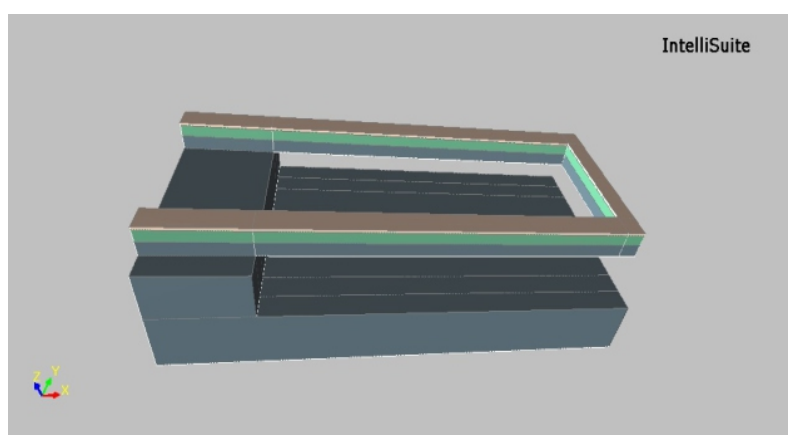

Fig. 2 U Shaped Cantilever

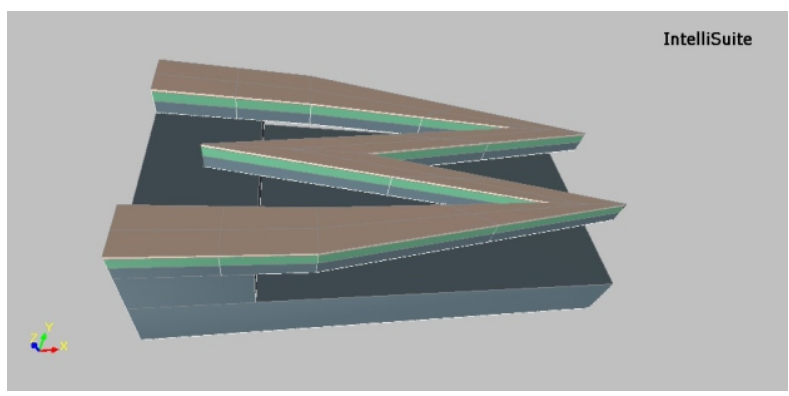

Fig. 3 W Shaped Cantilever 
of thermal imbalance in the processing, the beams are naturally deflected at relative humidity. This causes a change in surface stress and hence a change in the displacement. The sensitivity of these sensors increases when to change the length of the cantilever. The thickness of these layers and their particular coefficient of thermal expansion will establish the amount of deflection at a specific relative humidity. The second model of $U$ shaped cantilever is used with same as sensing layer. The geometry of thickness and materials must be selected so that the cantilevers will be flat at the maximum deflection of the cantilever beam. In third model Poly silicon is used as sensing layer. The thickness and materials should be preferred so that the cantilever beams will be even at the maximum deflection for the desired displacement.

\section{Simulation Results}

The mechanical study of the cantilever design was carried out in order to determine its modal displacement. In humidity sensor study was carried out using the thermo electromechanical (TEM) module of the Intellisuite. Here, the static displacement study was done on the cantilever beam. The outcome of result shows the displacement of the beam structure varied at different level. When the cantilever beam is weighted it moves in the Z-direction. Thus, the displacement study of the beam is done in the Z-direction. The $\mathrm{W}$ shaped cantilever beam showed a maximum displacement of 26.75 When the relative humidity of $100 \% \mathrm{RH}$. The result of the displacement, Mises stress and sensitivity analyses are shown in the Fig. 4 to 9.

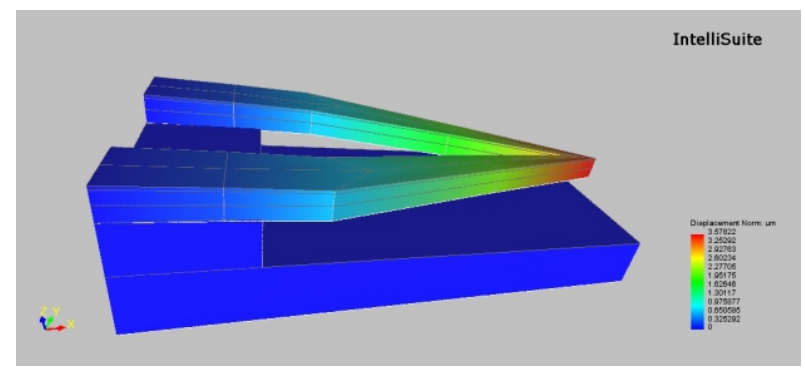

Fig. 4 Displacements at $50 \% \mathrm{RH}$ of V shaped cantilever

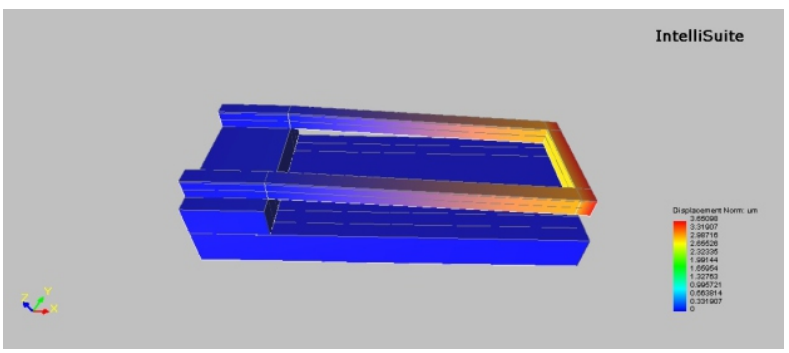

Fig. 5 Displacements at $50 \% \mathrm{RH}$ of $\mathrm{U}$ shaped cantilever

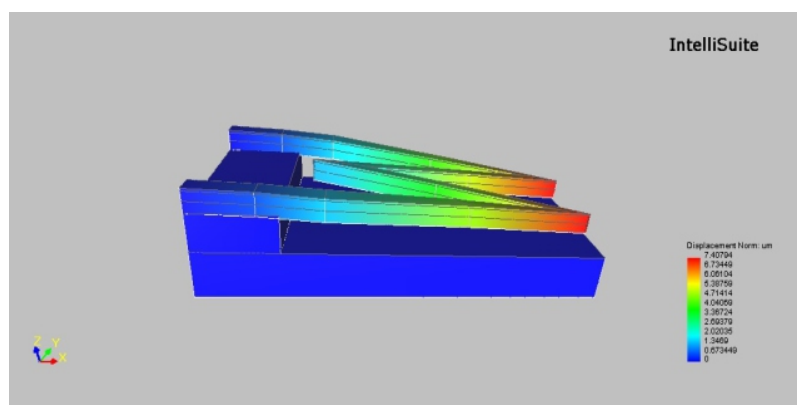

Fig. 6 Displacements at 50\%RH of W shaped cantilever

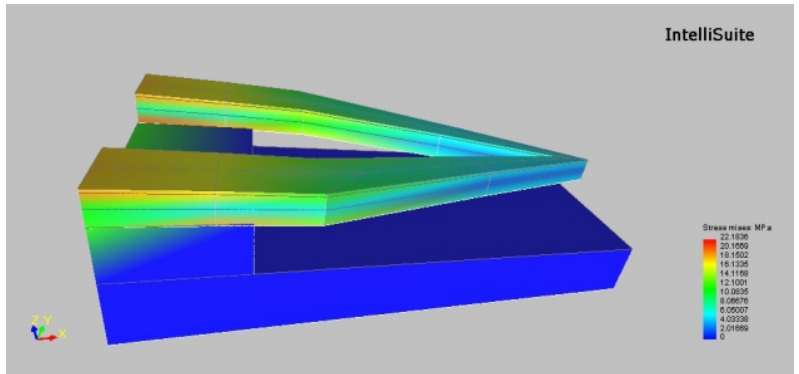

Fig. 7 Mises Stress at $30 \%$ RH of V shaped cantilever

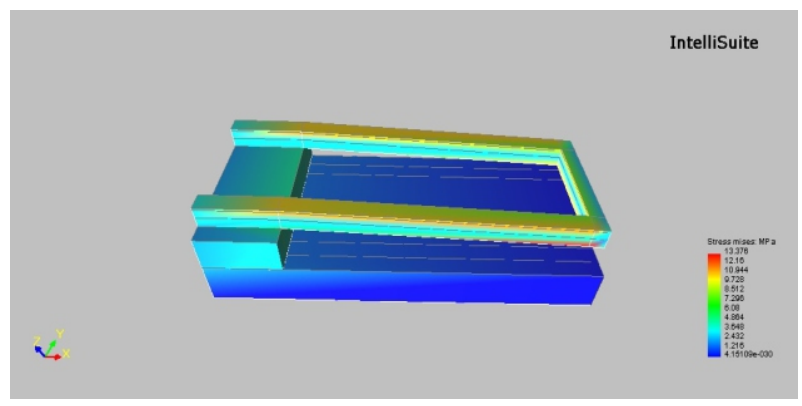

Fig. 8 Mises Stress at $30 \%$ RH of U shaped cantilever

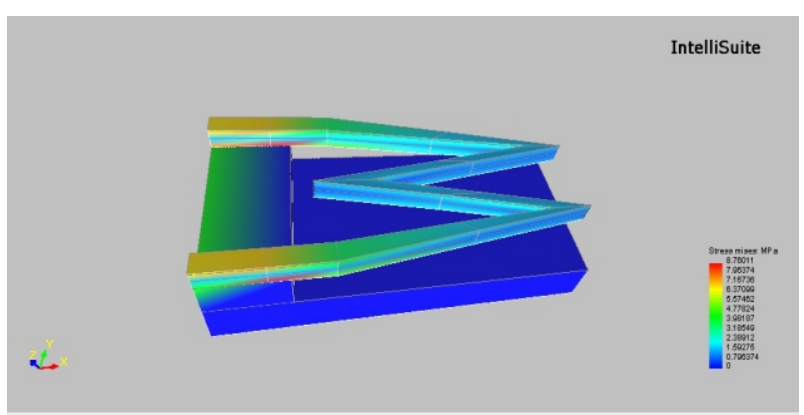

Fig. 9 Mises Stress at 10\%RH of W shaped cantilever 


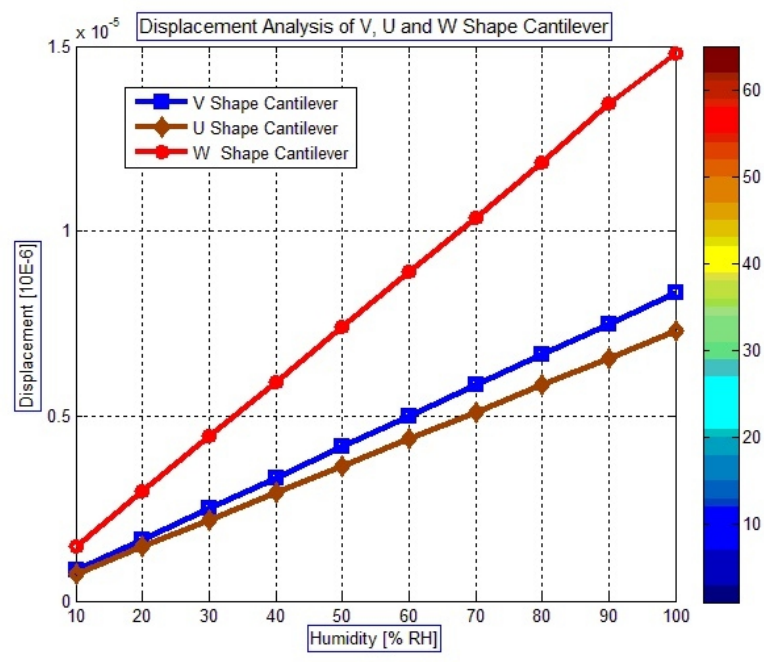

Fig .10 Displacement analysis of various cantilever

W shaped cantilever results are shown the maximum displacement of the MEMS cantilever structure. Then the Maximum stress is noted in Fig. 11 where W shaped cantilever has more Mises stress and comparison results of sensitivity also obtained that the $\mathrm{W}$ shaped cantilever sensors are summarized as shown in Fig.12. Hence, the $\mathrm{W}$ shape cantilever results are obtained the maximum displacement, maximum stress and sensitivity of the MEMS cantilever structure. The results show that both the displacement and sensitivity are increased with the change in beam sensing material or otherwise we can change in beam length, width and area.

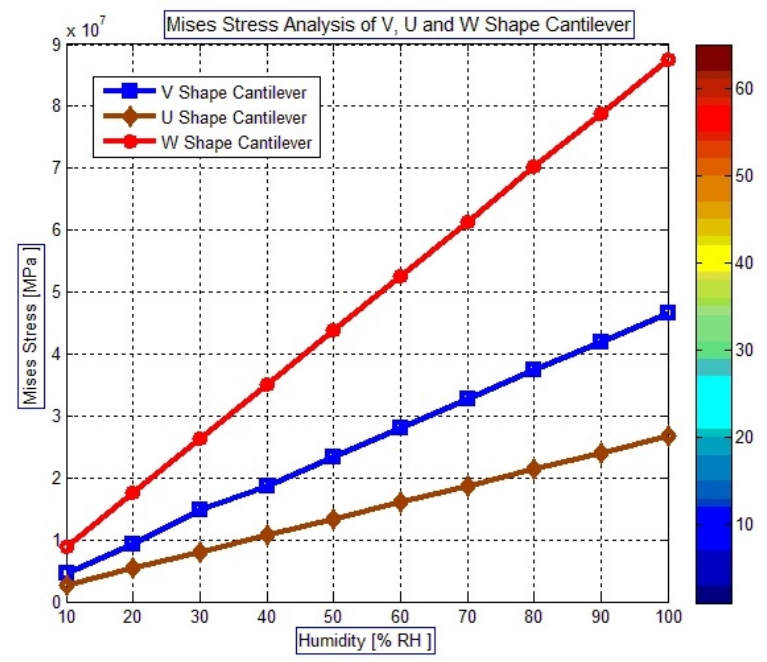

Fig .11 Mises Stress analysis of various cantilever

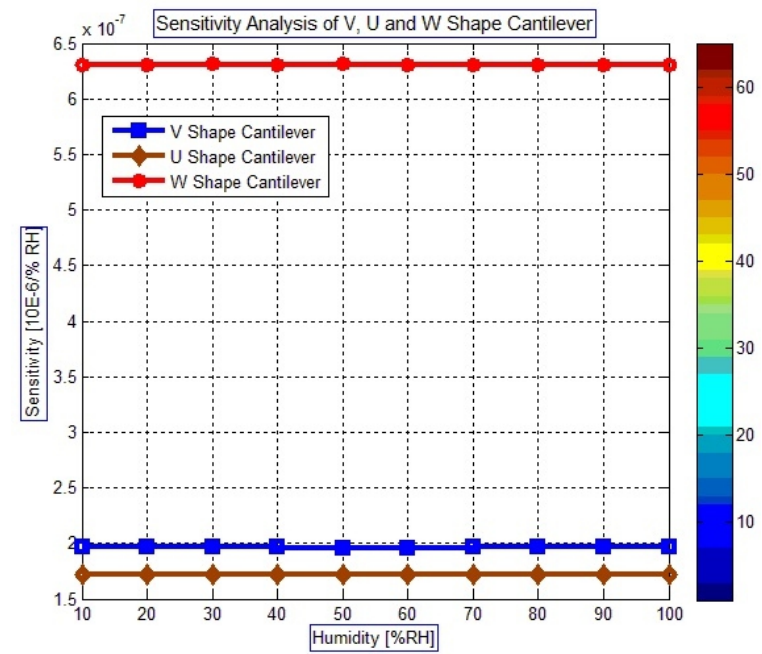

Fig .12 Sensitivity analysis of various cantilever

\section{Conclusion}

We have proposed an optimized various shapes of a cantilever with improved performance parameters such as displacement, maximum stress and sensitivity. These cantilevers have been designed and their sensitivity is also analyzed with different input pressure equivalent of humidity. We found that the micro cantilever humidity sensor exhibits good performance in the range of $10 \% \mathrm{RH}$ to $100 \%$ RH exponentially. Hence we conclude that the W shaped micro cantilever with a sensing layer of Polyimide has a more suitable for humidity sensor.

\section{Acknowledgment}

The authors would like to thank NPMASS (National Program on Micro and Smart Systems), Ministry of Defense, Government of India for providing the software facilities and support at Pondicherry University, Pondicherry.

\section{References}

[1] V. L. Nickolay, et al, "Cantilever transducers as a platform for chemical and biological sensors", AIPReview of Scientific Instruments, vol. 75, no. 7, July 2004, p. 2229-2253.

[2] S. Chatzandroulis , A. Tserepi, D. Goustouridis, P.Normand, D. Tsoukalas "Fabrication of single crystal Si cantilevers using a dry release processand application in a capacitive- type humidity sensor", Elsevier Science, Microelectronic Engineering, 2002, p. 955-961.

[3] B. Gardiner, "Murmurs of earth", Wired, Jun. 2013, p. $148-155$.

[4] Anja Boisen, Soren Dohn, Stephan Sylvest Keller, 
Silvan Schmid and Maria Tenje "Cantilever-like micromechanical sensors", Denmark, IOP Publishing Ltd printed in the UK \& the USA, 2011.

[5] R. Nicole, et al,"Humidity Sensors Principle, Mechanism, and Fabrication Technologies: A Comprehensive Review", Sensors , 2014, p.78817939.

[6] Lung-Tai Chen et el, "MEMS-based humidity sensor with integrated temperature compensation mechanism", Sensors and Actuators A 147 2008, p.522-528.
[7] J. Brugger, et al., "Microfabricated ultrasensitive piezoresistive cantilevers for torque magnetometry", Sensors Actuators, vol.73, 1999, p. 235-242.

[8] A. Nallathambi et al., "Performance analysis of cantilever based MEMS sensor for environmental applications ", Proc. International Conference on Smart Structures \& Systems, ISSS-2014, Chennai, INDIA, IEEE, 2014, p.118-122.

[9] A. Nallathambi et al. "A Cantilever Based MEMS Sensor for Detection of Greenhouse Gases", IOJER, vol 1, no. 2, 2015, p.16-21. 